\title{
Retrospective analysis of endocarditis patients to investigate the eligibility for oral antibiotic treatment in routine daily practice
}

\author{
J. C. Vroon - O. C. D. Liesdek · C. H. E. Boel · J. E. Arends · F. A. Niessen · H. C. van Heusden · M. J. Cramer • \\ T. I. G. van der Spoel · S. A. J. Chamuleau
}

Published online: 17 September 2020

(C) The Author(s) 2020

\begin{abstract}
Background According to the current guidelines of the European Society of Cardiology, patients with leftsided infective endocarditis are treated with intravenous antibiotics for 4-6 weeks, leading to extensive hospital stay and high costs. Recently, the Partial Oral Treatment of Endocarditis (POET) trial suggested that partial oral treatment is effective and safe in selected patients. Here, we investigated if such patients are seen in our daily clinical practice.

Methods We enrolled 119 adult patients diagnosed with left-sided infective endocarditis in a retrospective, observational study. We identified those that
\end{abstract}

\section{Electronic supplementary material The online version of this article (https://doi.org/10.1007/s12471-020-01490-2) contains supplementary material, which is available to authorized users.}

J. C. Vroon · H. C. van Heusden · M. J. Cramer Department of Cardiology, University Medical Center Utrecht, Utrecht, The Netherlands

\section{O. C. D. Liesdek}

Department of Cardiothoracic Surgery, University Medical Center Utrecht, Utrecht, The Netherlands

\section{H. E. Boel · F. A. Niessen}

Department of Microbiology, University Medical Center Utrecht, Utrecht, The Netherlands

\section{J. E. Arends}

Department of Internal Medicine and Infection Diseases, University Medical Center Utrecht, Utrecht, The Netherlands

T. I. G. van der Spoel

Department of Cardiology, Haga Hospital, The Hague, The Netherlands

\section{S. A. J. Chamuleau ( $\triangle)$}

Department of Cardiology, Amsterdam University Medical Center, AMC/University of Amsterdam, Amsterdam, The Netherlands

s.a.j.chamuleau@amsterdamumc.nl would be eligible for switching to partial oral antibiotic treatment as defined in the POET trial (e.g. stable clinical condition without signs of infection). Secondary objectives were to provide insight into the time until each patient was eligible for partial oral treatment, and to determine parameters of longer hospital stay and/or need for extended intravenous antibiotic treatment.

Results Applying the POET selection criteria, the condition of 38 patients (32\%) was stable enough to switch them to partial oral treatment, of which 18 (47.3\%), 8 (21.1\%), 9 (23.7\%) and 3 patients $(7.9 \%)$ were eligible for switching after 10, 14, 21 days or 28 days of intravenous treatment, respectively.

Conclusion One-third of patients who presented with left-sided endocarditis in routine clinical practice were possible candidates for switching to partial oral treatment. This could have major implications for both the patient's quality of life and healthcare costs. These results offer an interesting perspective for implementation of such a strategy, which should

\section{What's new?}

- Partial oral treatment is associated with favourable outcomes, low hospital mortality, a low reinfection rate and cost savings.

- One-third of 119 patients with left-sided infective endocarditis were in a clinically stable condition and eligible for switching to partial oral treatment, according to the criteria of the previously published Partial Oral Treatment of Endocarditis trial.

- Our study offers a perspective for implementation of this strategy, accompanied by a prospective cost-effectiveness analysis. 
be accompanied by a prospective cost-effectiveness analysis.

Keywords Endocarditis · Cardiac surgery · Oral treatment $\cdot$ Antibiotic treatment

\section{Introduction}

Currently, all patients with left-sided infective endocarditis are treated with intravenous antibiotics for 4-6 weeks, according to the guidelines of the European Society of Cardiology [1-3]. Adequate management of this fatal disease, with an in-hospital mortality rate of $15-40 \%$ [2-7], comprises early diagnosis with early initiation of intravenous bacteria-specific antibiotic treatment and surgical intervention if needed. The paradigm that patients with infective endocarditis must be treated with intravenous antibiotics under continuous clinical surveillance is changing due to new emerging evidence [1, 4], with a potential for partial outpatient treatment-intravenously or even orally.

Typically, intravenous treatment for 4-6 weeks requires prolonged hospital stay or a home-care management system. In additional, this treatment is associated with a reduced patient's quality of life, higher risk of hospital-acquired complications resulting in deterioration of the patient's clinical status, and increased healthcare costs $[4,5,8,9]$ Due to these disadvantages, interest has been raised in converting in-hospital intravenous antibiotic treatment to outpatient parenteral treatment in clinically stable patients 2 weeks after initiation of the intravenous treatment. Several studies have shown outpatient parenteral treatment is an effective and, importantly, safe option [10-14]. Furthermore, outpatient parenteral treatment reduces the risk of hospital-acquired complications, the psychological burden and the recovery time, and thus in-hospital costs [13-17]. Still, outpa- tient treatment requires practical home care and close monitoring of the therapeutic effects and side effects of the therapy. These logistic issues require dedicated care by home-visit nursing staff $[1,3,4]$.

The drawbacks of outpatient antibiotic treatment for endocarditis can potentially be overcome by the introduction of a convenient alternative: partial oral treatment. Recently, Iversen et al. conducted a randomised controlled trial, the Partial Oral Treatment of Endocarditis (POET) trial, and reported that partial oral antibiotic treatment is non-inferior to intravenous therapy in patients in stable clinical condition [4].

Based on these promising results, we retrospectively evaluated patients diagnosed with left-sided infective endocarditis who presented to the University Medical Center Utrecht from 1 January 2016 until 1 December 2018. The main objective was to determine the number of patients in daily clinical practice that fulfil the POET criteria for clinically stable condition, making them eligible for safe partial oral antibiotic treatment.

\section{Methods \\ Study design and population}

In this retrospective, observational study, we reviewed the patient files of 119 consecutive adult patients with infective endocarditis that were discussed in the multidisciplinary endocarditis team; they received inpatient treatment at the University Medical Center Utrecht, the Netherlands and fulfilled the Duke criteria (definite or possible endocarditis) from 1 January 2016 until 1 December 2018.

To evaluate which patients would have been, hypothetically, in stable clinical condition to qualify for oral treatment, we assessed them using the selection criteria used in the earlier POET trial of Iversen et al.:
Fig. 1 Flowchart displaying four criteria to identify patients in clinically stable condition to qualify for switching to partial oral treatment. A total of 38 patients fulfilled all criteria, while14 patients failed to meet the fourth criterion due to other indications for intravenous antibiotics. (UMCU University Medical Center Utrecht)

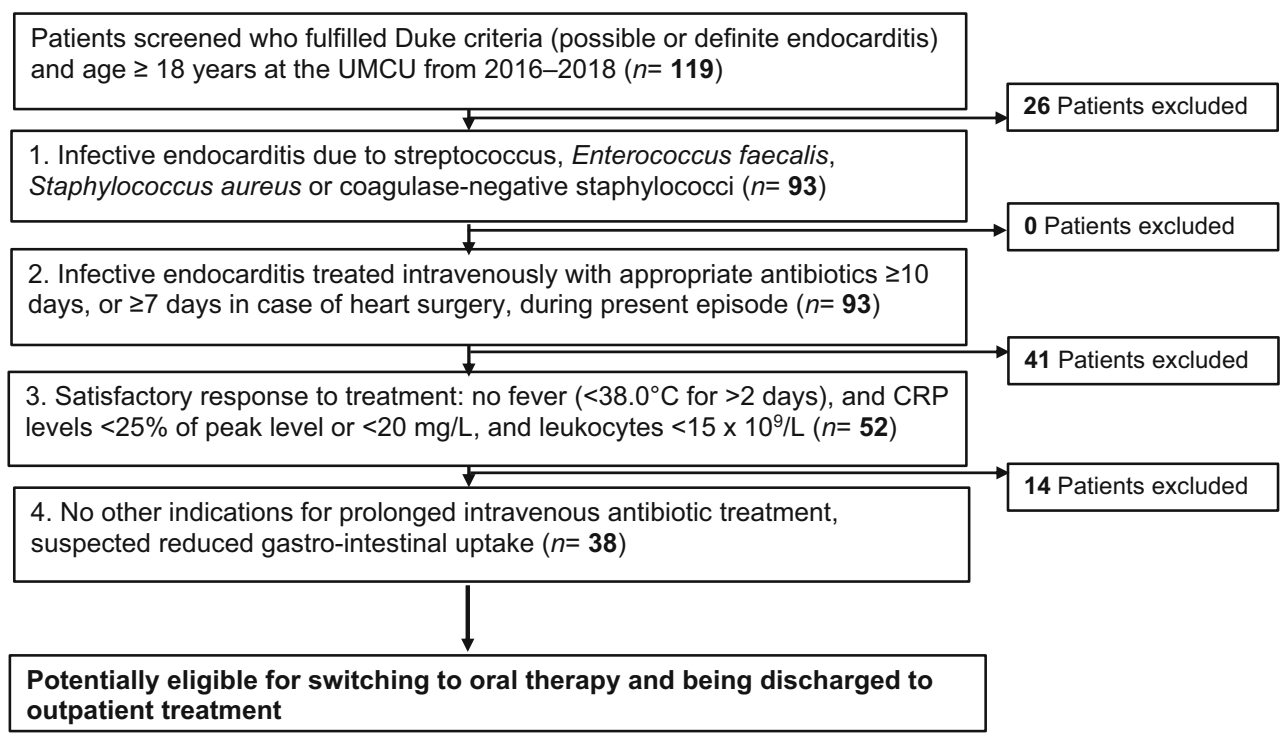


Table 1 Characteristics of patients with left-sided infective endocarditis

\begin{tabular}{|l|c|}
\hline Variable & Patients $(n=119)$ \\
\hline Age, years (mean \pm SD) & $64.8 \pm 13.1$ \\
\hline Female gender & $31(26.1)$ \\
\hline Comorbidity & \\
\hline - Diabetes & $20(16.8)$ \\
\hline - COPD/asthma & $9(7.6)$ \\
\hline - Renal failure & $7(5.9)$ \\
\hline - Cancer & $29(24.4)$ \\
\hline Infective endocarditis according to Duke criteria \\
\hline - Possible & $25(21.0)$ \\
\hline - Definite & $94(79.0)$ \\
\hline Duke criteria & \\
\hline - Pathological criteria & $51(42.9)$ \\
\hline - Major & \\
\hline a. Positive blood culture & $78(65.5)$ \\
\hline b. Positive imaging & $102(85.7)$ \\
\hline - Minor & \\
\hline a. Predisposition (HC or IV) & $52(43.7)$ \\
\hline b. Fever & $79(66.4)$ \\
\hline C. Vascular phenomena & $22(18.5)$ \\
\hline d. Immunological phenomena & $4(3.4)$ \\
\hline e. Microbiological evidence & $31(26.1)$ \\
\hline Microorganisms & \\
\hline - Streptococcus & $43(36.1)$ \\
\hline - Enterococcus faecalis & $17(14.3)$ \\
\hline - Staphylococcus aureus & $25(21.0)$ \\
\hline - Coagulase-negative staphylococci & $8(6.7)$ \\
\hline Laboratory results at admission (mean $\pm S D)$ & \\
\hline - Hemoglobin, mmol/L & $116.1 \pm 1.3$ \\
\hline - CRP, mg/L & \\
\hline - Leukocytes, $\times 109 / L$ & \\
\hline - Creatinine, $\mu$ mol/L & \\
\hline
\end{tabular}

(1) positive blood cultures for the most commonly cultured microorganisms (i.e. streptococcus, Enterococcus faecalis, Staphylococcus aureus and coagulasenegative staphylococci); (2) patients had to be treated with intravenous antibiotics for at least 10 days or for at least 7 days after surgery; (3) evident clinical and biochemical response to the antibiotic therapy, i.e. no fever for at least 2 days consecutively, and a decrease in C-reactive protein (CRP) levels below $25 \%$ of the peak CRP level at admission or CRP $<20 \mathrm{mg} / \mathrm{L}$ and leukocytes $<15 \times 10^{9} / \mathrm{L}$; and (4) absence of other indications for intravenous antibiotic treatment, such as pneumonia, or prolonged hospital stay due to complications. A flowchart is depicted in Fig. 1.

\section{Data collection and outcomes}

We obtained all data and patient characteristics from the patient files. The primary outcome was the number of clinically stable patients that would have been
Table 1 (Continued)

\begin{tabular}{l|c}
\hline $\begin{array}{l}\text { Variable } \\
\text { Pre-existing device }\end{array}$ & Patients $(n=119)$ \\
\hline - Prosthetic heart valve & $48(40.3)$ \\
\hline - Pacemaker & $13(10.9)$ \\
\hline - Other known valve disease & \\
\hline a. Bicuspid valve & $5(4.2)$ \\
\hline b. Moderate or severe regurgitation & $50(42.0)$ \\
\hline Cardiac involvement (left) & \\
\hline - Mitral valve & $38(31.9)$ \\
\hline - Aortic valve & $47(39.5)$ \\
\hline - Mitral and aortic valve & $15(12.6)$ \\
\hline - Pacemaker endocarditis & $5(4.2)$ \\
\hline - Vegetation size $>9$ mm & $28(23.5)$ \\
\hline Surgery & $71(59.7)$ \\
\hline - EuroSCORE II (mean \pm SD) & $12.0 \pm 13.9$ \\
\hline Pacemaker lead extraction & $1(0.8)$ \\
\hline Mortality & $31(26.1)$ \\
\hline - <30 days after diagnosis & $14(45.2)$ \\
\hline - <1 year after diagnosis & $17(54.8)$ \\
\hline Mortality after surgery & $14(19.7)$ \\
\hline - <30 days after surgery & $7(50.0)$ \\
\hline$-<1$ year after surgery & $7(50.0)$ \\
\hline All data are $n$ (\%) unless stated otherwise & \\
\hline COPD chronic obstructive pulmonary disease, $H C$ heart condition, $I$ intra- \\
\hline venous drug use, CRPC-reactive protein & \\
\hline
\end{tabular}

eligible for switching to oral antibiotic treatment according to the criteria used in the recent POET trial. Secondary outcomes were: (1) evaluation of the individual time until a patient was eligible for partial oral treatment according to clinical and biochemical laboratory results; and (2) determination of other indications for longer hospital stay or intravenous antibiotic treatment due to complications.

\section{Results}

A total of 119 adult patients with left-sided infective endocarditis were included, of whom $79 \%$ fulfilled the modified Duke criteria for definite endocarditis and $21 \%$ the criteria for possible endocarditis. Demographics and clinical characteristics of all patients are shown in Tab. 1.

The majority $(74 \%)$ was male and their mean age was 65 years. Streptococcus was the most frequently identified microorganism, causing endocarditis in 43 cases $(36 \%)$, followed by S. aureus in 25 patients (21\%); 26 patients were infected by another causative pathogen $(n=18)$ or had consistently negative blood cultures $(n=8)$. The mean ( \pm standard deviation) CRP level at admission was $123 \mathrm{mg} / \mathrm{L}( \pm 105)$ and the mean leukocyte count was $15.5 \times 10^{\%} / \mathrm{L}( \pm 25.3)$. The aortic valve was affected in 47 patients and the mitral valve in 38 patients; in 15 patients, both the aortic and mitral valve were infected. In 14 patients, imag- 
ing was inconclusive, and they were diagnosed with endocarditis based on other Duke criteria.

Thirty of the 48 patients with a previously inserted prosthetic heart valve presented with prosthetic valve endocarditis. Five out of 13 patients with an implanted pacemaker were diagnosed with active pacemaker lead endocarditis; one of these 5 patients underwent a lead extraction. All 13 patients with or without pacemaker lead endocarditis were diagnosed by transthoracic echocardiography. Additional imaging consisted of transoesophageal echocardiography and/or positron emission tomography combined with computed tomography (PET-CT) in case of inconclusive transthoracic echocardiography. Approximately $60 \%(n=71)$ of all included patients underwent cardiac surgery; their mean EuroSCORE II was $12 \%$.

The overall mortality was $26.1 \%(n=31)$, of which $45.2 \%(n=14)$ died within 30 days after endocarditis was diagnosed and $54.8 \%(n=17)$ within 1 year after diagnosis. A total of 14 patients (19.7\%) died after surgery, of whom 7 died within 30 days after surgery and 7 within one year after surgery. According to the selection criteria, as depicted in Fig. 1, a group of 93 patients fulfilled the criterion of infection with an endocarditis-causing microorganism. All these patients were intravenously treated with antibiotics for at least 10 days during conservative treatment or for at least 7 days after surgery. Antimicrobial therapy was in all cases initiated on the same day endocarditis was diagnosed.

Of the 93 patients who fulfilled the first selection criterion, 52 (56\%) had a sufficient clinical and biochemical response to therapy according to the third criterion. Eventually 38 patients (32\% of 52 ) had no other indication for intravenous antibiotics or prolonged hospital stay and were clinically stable and ready to convert to oral treatment of the infective endocarditis (Fig. 1). After 10, 14 and 21 days of intravenous antibiotic treatment, 18 patients $(47.3 \%), 8$ patients $(21.1 \%)$ and 12 patients $(31.6 \%)$, respectively, were eligible for POET (Tab. 2; see also Electronic Supplementary Material). Of the 52 patients, $14 \mathrm{did}$ not fulfil the final POET criterion, since they had com-

Table 2 Patients with endocarditis eligible for partial oral antibiotic treatment

\begin{tabular}{|c|c|}
\hline Criterion & Patients $(n=38)$ \\
\hline No fever ${ }^{\mathrm{a}}$ and CRP levels $<25 \%$ of peak level ${ }^{\mathrm{b}}(n)$ & 19 \\
\hline $\begin{array}{l}\text { No fever }{ }^{\mathrm{a}} \text { and } \mathrm{CRP}<20 \mathrm{mg} / \mathrm{L} \text { and leukocytes } \\
<15 \times 10^{9} / \mathrm{L}(n)\end{array}$ & 19 \\
\hline \multicolumn{2}{|l|}{ Days after starting intravenous treatment $(n(\%))$} \\
\hline - $10-13$ days & $18(47.3)$ \\
\hline - 14-20 days & $8(21.1)$ \\
\hline$-21-27$ days & $9(23.7)$ \\
\hline$-28-33$ days & $3(7.9)$ \\
\hline $\begin{array}{l}\text { CRPC-reactive protein } \\
\text { aTemperature }<38^{\circ} \mathrm{C} \text { for at least } 2 \text { days } \\
\text { bPeak level is CRP level at day of admission }\end{array}$ & \\
\hline
\end{tabular}

Table 3 Patients with endocarditis ineligible for partial oral antibiotic treatment ${ }^{\mathrm{a}}$

\begin{tabular}{|c|c|}
\hline Criterion & Patients $(n=14)$ \\
\hline \multicolumn{2}{|c|}{ Endocarditis-related complications during treatment } \\
\hline - Severe MI (reoperation) & 1 \\
\hline - Infected knee prosthesis & 1 \\
\hline \multicolumn{2}{|l|}{ Complications after surgery } \\
\hline - Acute renal failure (CVVH) & 4 \\
\hline - Epileptic insult & 1 \\
\hline - Pneumonia/pneumothorax & 4 \\
\hline - SAB/mycotic aneurysm & 1 \\
\hline - Mediastinitis & 1 \\
\hline \multicolumn{2}{|l|}{ Other indication for hospitalization } \\
\hline - Continual bleeding/factor VIII deficiency & 1 \\
\hline - Epidural abscess & 1 \\
\hline $\begin{array}{l}\text { - Unsafe swallowing function as result of } \\
\text { CVA }\end{array}$ & 1 \\
\hline - Delirium & 4 \\
\hline
\end{tabular}

plications requiring prolonged intravenous treatment and/or longer hospitalization (Tab. 3). Most common indications were acute renal failure and delirium.

\section{Discussion}

In this retrospective study, we observed that a significant group of patients with left-sided endocarditis in daily clinical practice (32\%) was eligible for partial oral treatment with antibiotics. We found a slightly higher percentage of patients qualifying as clinically stable than that in the POET trial $(32 \%$ vs $20 \%)$. The median time to switching to oral treatment in the initial POET trial was 17 days (range: 12-24) [4], which compares to our findings (mean time: 14 days; range: 10-33), although the range may be a matter of debate. However, it should be noted that 41 patients of the group of 93 did not have a sufficient clinical and biochemical response to the therapy. They were not in a clinically stable condition because of persistently high infection parameters caused by for example $S$. aureus bacteraemia or other infections, or patients died shortly after the diagnosis of endocarditis because of endocarditis-related complications during therapy.

Guidelines recommend intravenous antimicrobial therapy for 4-6 weeks [2, 3, 18, 19]. Intravenous medication is considered to be more effective than oral treatment, because therapeutic concentrations are rapidly achieved in the blood. In addition, most complications arise in the first 2 weeks-the so-called initial or critical phase-which require adequate intravenous treatment $[2-5,20]$. In our study, $21.8 \%$ of all endocarditis patients were in clinically stable condition and eligible for POET between 10 to 21 days 
after initiation of antibiotic therapy. However, a larger time frame should be considered. Consequently, by extending the time frame to 28 days, we identified an even larger group of clinically stable patients that may be eligible for switching to oral antibiotics (29.4\%), which is clinically highly relevant.

Other recommendations for oral treatment have been addressed by several studies. Earlier research confirmed that the outcomes of partial oral treatment are non-inferior to the outcomes of intravenous treatment [4]. Observational studies examined the efficacy of several oral antibiotics in the treatment of endocarditis. It has been suggested that switching to oral treatment is an effective and safe alternative [1, $17,19,21,22]$. In addition, it has been reported that switching to oral antibiotics is a feasible treatment, associated with favourable outcomes, low hospital mortality, a low reinfection rate, and cost savings. More prospective studies are crucial to evaluate the alternative oral therapy in practice and prove its costeffectiveness.

\section{POET in future research}

There are a few aspects of POET to be considered before conducting such studies, especially regarding the exclusion criteria, in an effort to include more patients. First, only patients with left-sided endocarditis were included; however, other studies also examined switching to oral treatment in patients with right-sided endocarditis. These studies reported efficient and safe treatment with oral ciprofloxacin and rifampicin without a higher risk of reinfection and with less drug toxicity [17, 19, 23, 24]. Although infective endocarditis most frequently affects the left side of the heart, right-sided endocarditis is seen on a regular basis. In our study, 5 patients were excluded due to the presence of right-sided infective endocarditis, while 3 of them were hypothetically ready for oral treatment. Also, for patients with right-sided endocarditis, partial oral treatment is highly relevant, and they should be included in future studies.

Second, 4 out of 8 infective endocarditis patients with persistently negative blood cultures in our study were in a clinically stable condition and eligible for POET within 16 days after initiation of intravenous antibiotic treatment. No previous study has investigated oral antibiotic treatment of infective endocarditis patients with persistently negative blood cultures. Moreover, even now, the best choice of specific intravenous antibiotic treatment is not clear, due to scarce data in this vulnerable patient population. Despite the good clinical condition of these patients, proposing switching to oral treatment would result in too much uncertainty during treatment.

Finally, it is important to note that the antibiotic regimens in the POET trial are not commonly used in the Netherlands. Although it is necessary to adhere to the adage of using two antibiotics with high biological availability, other antibiotics will be chosen for the conversion to oral treatment, in consultation with the guidelines of the Dutch Working Party on Antibiotic Policy (Stichting Werkgroep Antibiotica Beleid).

\section{Study limitations}

Our study has some limitations, especially related to the design of an observational, retrospective study. After trying to obtain all data, those of 9 patients were still missing. The missing data concerned CRP levels, leukocyte counts and body temperature. Due to the referral of patients to our hospital and the discharge to another hospital, no continuous data were directly available. These 9 patients were lost to follow up after the third criterion of the flowchart.

\section{Conclusion}

This retrospective, observational study showed that one-third of patients who presented with left-sided endocarditis in daily clinical practice were possible candidates for switching to partial oral treatment. Obviously, this could have major implications for both the patient's quality of life and healthcare costs. These results offer an interesting perspective for implementation of such a strategy, which should be accompanied by a proper prospective cost-effectiveness analysis.

Acknowledgements We would like to thank all members of the endocarditis team at the University Medical Center Utrecht.

Conflict of interest J.C. Vroon, O.C.D. Liesdek, C.H.E. Boel, J.E. Arends, F.A. Niessen, H.C. van Heusden, M.J. Cramer, T.I.G. van der Spoel and S.A.J. Chamuleau declare that they have no competing interests.

Open Access This article is licensed under a Creative Commons Attribution 4.0 International License, which permits use, sharing, adaptation, distribution and reproduction in any medium or format, as long as you give appropriate credit to the original author(s) and the source, provide a link to the Creative Commons licence, and indicate if changes were made. The images or other third party material in this article are included in the article's Creative Commons licence, unless indicated otherwise in a credit line to the material. If material is not included in the article's Creative Commons licence and your intended use is not permitted by statutory regulation or exceeds the permitted use, you will need to obtain permission directly from the copyright holder. To view a copy of this licence, visit http://creativecommons.org/licenses/by/4.0/.

\section{References}

1. Iversen K, Høst N, Bruun NE, et al. Partial oral treatment of endocarditis. Am Heart J.2013;165:116-22.

2. Baddour LM, Wilson WR, Bayer AS, et al. Infective endocarditis in adults: diagnosis, antimicrobial therapy, and management of complications. Circulation. 2015;132:1435-86. 
3. Habib G, Lancellotti P, Antunes MJ, et al. 2015 ESC infective endocarditis. Eur Heart J. 2015;3:3075-128.

4. Iversen K, Ihlemann N, Gill SU, et al. Partial oral versus intravenous antibiotic treatment of endocarditis. N Engl J Med. 2018;380:415-24.

5. CahillTJ, Baddour LM, HabibG, etal. Challengesininfective endocarditis. J Am Coll Cardiol. 2017;69:325-44. https:// doi.org/10.1016/j.jacc.2016.10.066.

6. AbegazTM, BahagavathulaAS, Gebreyohannes EA, Mekonnen AB, Abebe TB. Short- and long-term outcomes in infective endocarditis patients: a systematic review and metaanalysis. BMCCardiovasc Disord. 2017;17:291. https://doi. org/10.1186/s12872-017-0729-5.

7. van den Brink FS, Swaans MJ, Hoogendijk MG, et al. Increased incidence of infective endocarditis after the 2009 European society of cardiology guideline update: a nationwide study in the Netherlands. Eur Heart J Qual Care Clin Outcomes. 2017;3:141-7.

8. Wind J, Polle SW, Fung Kon Jin PHP, et al. Systematic review of enhanced recovery programmes in colonic surgery. Br J Surg. 2006;93:800-9.

9. Klein M, Wang A. Infective endocarditis. J Intensive Care Med. 2016;31:151-63.

10. Partridge DG, O'Brien E, Chapman ALN. Outpatient parenteral antibiotic therapy for infective endocarditis: a review of 4 years' experience at a UK centre. Postgrad Med J. 2012;88:377-81.

11. Gil-Navarro MV, Lopez-Cortes LE, Luque-Marquez R, Galvez-Acebal J, de Alarcon-Gonzalez A. Outpatient parenteral antimicrobial therapy in Enterococcus faecalis infective endocarditis. JClin Pharm Ther. 2018;43:220-3.

12. Amodeo MR, Clulow T, Lainchbury J, et al. Outpatient intravenous treatment for infective endocarditis: safety, effectiveness and one-year outcomes. J Infect. 2009;59:387-93.

13. Lacroix A, Revest M, Patrat-Delon S, et al. Outpatient parenteral antimicrobial therapy for infective endocarditis: a cost-effective strategy. Med Mal Infect. 2014;44:327-30.

14. McMahon JH, O'keeffe JM, The Victorian Hith Outcomes Study Group, Grayson ML. Is hospital-in-the-home (HITH) treatment of bacterial endocarditis safe and effective? Scand J Infect Dis. 2008;40:40-3.
15. Berg SK, Preisler P, Pedersen BD. Patients perspective on endocarditis-an intermezzo in life. Eur J Cardiovasc Nurs. 2010;9:126-31.

16. de Saint-Hubert M, Schoevaerdts D, Poulain G, Cornette P, Swine C. Risk factors predicting later functional decline in older hospitalized patients. Acta Clin Belg. 2009;64:187-94.

17. Al-Omari A, Cameron DW, Lee C, Corrales-Medina VF. Oral antibiotic therapy for the treatment of infective endocarditis: a systematic review. BMC Infect Dis. 2014;14:140.

18. Baddour LM, Wilson WR, Bayer AS, et al. Infective endocarditis: diagnosis, antimicrobial therapy, and management of complications a statement for healthcare professionals from the committee on rheumatic fever, endocarditis, and Kawasaki disease, council on cardiovascular disease in the young, and the councils on clinical cardiology, stroke, and cardiovascular surgery and anesthesia, American heart association: endorsed by the infectious diseases society of America. Circulation. 2005;111:394-43.

19. Mzabi A, Kernéis S, Richaud C, Podglajen I, FernandezGerlinger MP, Mainardi JL. Switch to oral antibiotics in the treatment of infective endocarditis is not associated with increased risk of mortality in non-severely ill patients. Clin Microbiol Infect. 2016;22:607-12.

20. Murdoch DR, Corey RG, Hoen B, et al. Clinical presentation, etiology, and outcome of infective endocarditis in the 21st century: the international collaboration on endocarditis-prospective cohort study. Arch Intern Med. 2009;169:463-73.

21. Colli A, Campodonico R, Gherli T. Early switch from vancomycin to oral linezolid for treatment of gram-positive heart valve endocarditis. Ann Thorac Surg. 2007;84:87-91.

22. Demonchy E, Dellamonica P, Roger PM, Bernard E, Cua E, Pulcini C. Audit of antibiotic therapy used in 66 cases of endocarditis. Med Mal Infect. 2011;41:602-7.

23. Heldman AW, Hartert TV, Ray SC, et al. Oral antibiotic treatment of right-sided staphylococcal endocarditis in injection drug users: prospective randomized comparison with parenteral therapy. Am J Med. 1996;101:68-76.

24. Dworkin RJ, Sande MA, Lee BL, Chambers HF. Treatment of right-sided staphylococcus aureus endocarditis in intravenous drug users with ciprofloxacin and rifampicin. Lancet. 1989;2:1071-3. 